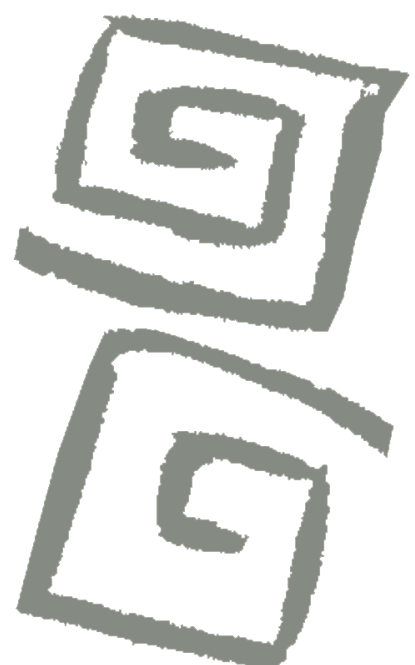

\title{
Ética del cuidado y atención pública en salud mental: un estudio de caso en Barcelona
}

\author{
Ethics of care and public mental health care: a case \\ study in Barcelona
}

Jaume Martínez Flores ${ }^{1}$, Margot Pujal i Llombart ${ }^{2}$, Enrico Mora ${ }^{3}$

\begin{abstract}
${ }^{1}$ Autor de correspondencia. Magíster en Investigación e Intervención Psicosocial. Investigador, Grupo Lis, Grupo de Investigación Des-Subjectant, Universitat Autónoma de Barcelona, Barcelona, España. $₫$ iD

${ }^{2}$ Doctora en Psicología Social. Profesora Titular, Facultad de Psicología, Universitat Autónoma de Barcelona, Barcelona, España. $\triangle$ iD

${ }^{3}$ Doctor, Profesor Agregado, Facultad de Ciencias Políticas i de Sociología, Universitat Autónoma de Barcelona, Barcelona, España. $\bowtie$ iD
\end{abstract}

RESUMEN El presente artículo tiene como objetivo analizar, desde la perspectiva de género, los servicios de atención pública en salud mental en la ciudad de Barcelona, a través de un estudio de caso: el servicio de salud mental de personas adultas en el contexto de la última reforma en salud mental de orientación comunitaria y transdisciplinaria. Desde un abordaje cualitativo, estudiamos los diferentes discursos que las y los profesionales en salud mental elaboran sobre cómo abordan la relación terapéutica entre profesional y persona usuaria y, de forma específica, la vulnerabilidad y las propuestas de respuesta. Entre noviembre de 2018 y abril de 2019, se realizaron tres grupos de discusión con profesionales y un grupo conformado por mujeres usuarias. Sobre los datos obtenidos se realizó análisis de contenido categorial. Los resultados muestran una tendencia a entender y abordar la vulnerabilidad desde la ética del cuidado a partir de un modelo de atención comunitaria, que diferencia los discursos entre los hombres y las mujeres que han participado en la investigación. Concluimos que el modelo comunitario, al basarse en la interdependencia, promueve la recuperación de la ciudadanía de las personas usuarias, contraponiéndose al modelo hospitalario.

PALABRAS CLAVES Salud Mental; Centros Comunitarios de Salud Mental; Investigación Cualitativa; Género y Salud; Vulnerabilidad Social; España.

\begin{abstract}
This article aims to analyze public mental health services in the city of Barcelona from a gender-based perspective. We do so through a case study of mental health services for adults in the context of recent community-oriented and transdisciplinary mental health care reforms. Employing a qualitative methodology, we look at the discourses of mental health professionals regarding the ways in which they approach the therapeutic relationship between professional and user, specifically with reference to vulnerability and proposals for addressing it. Between November 2018 and April 2019 we conducted three discussion groups with professionals and another with a group of female users. Data analysis was guided by a categorical content analysis model. The results show a tendency to understand and address vulnerability from an ethics of care based on a community care model, with differences in the discourses of men and women who participated in the research. We conclude that the community-based model promotes the recovery of users' citizenship, as it is based on a notion of interdependence, in contrast to the hospital-based mental health model.
\end{abstract}

KEY WORDS Mental Health; Community Mental Health Centers; Qualitative Research; Gender and Health; Social Vulnerability; Spain. 


\section{INTRODUCCIÓN}

Esta investigación se sitúa en la encrucijada del neoliberalismo y el patriarcado en un ámbito muy concreto: la atención feminizada en salud mental en el contexto español y catalán de la reforma en salud mental de las dos últimas décadas. Desde una perspectiva crítica, nos proponemos explorar cómo estos dos procesos históricos se manifiestan en los diferentes agentes que se encuentran en dicho contexto. Para ello centramos nuestra atención en el estudio de la ética del cuidado como forma de relacionarse en el ámbito de la salud mental.

Este artículo se enmarca dentro de un proyecto más amplio titulado La atención en salud mental desde una perspectiva de género: organización, gestión y subjetividad en el patriarcado neoliberal, dirigido por el Dr. Enrico Mora y la Dra. Margot Pujal, en el marco de los grupos de investigación LisEstudis Socials i de Gènere sobre la corporalitat, la subjetivitat $i$ el patiment evitale, y el grupo Des-Subjectant, de la Universitat Autònoma de Barcelona.

A pesar de la tendencia de la última reforma en salud mental de orientación comunitaria y multidisciplinar ${ }^{(1)}$, los y las profesionales de la salud mental experimentan como colectivo una fuerte tensión en su ámbito laboral, que se ha traducido en un deterioro de su salud $d^{(2,3)}$ y en malestar emocional. Por un lado, los servicios que orientan los centros de salud mental están destinados a incrementar el bienestar de las personas que acuden a él, cuya demanda no ha cesado de aumentar. A pesar de ello, los recursos que necesitan no se han visto sustancialmente modificados. Según la Organización Mundial de la Salud $(\mathrm{OMS})^{(4,5,6)}$, se estima a nivel mundial una brecha del $11 \%$ entre la carga de los trastornos neuropsiquiátricos (13\% de los costos globales en salud), y el presupuesto de salud mental destinado (que es del $2 \%$ ), y el pronóstico es que una de cada cuatro personas en el mundo experimentará un trastorno mental en algún momento de su vida, siendo una de las principales causas de discapacidad en el mundo. Por el otro, se han introducido modelos de gestión empresarial de los servicios en salud mental, conocidos como el New Public Management ${ }^{(7,8,9)}$. La atención clínica y de gestión se apoya en distintos modelos (por ejemplo, comunitario, hospitalario o atención domiciliaria) que conllevan experiencias muy diferentes, tanto para las y los profesionales como para las personas usuarias ${ }^{(10)}$. Los modelos de atención en salud mental que se basan en presupuestos epistemológicos distintos, que van desde concepciones psicodinámicas y psicosociales de las personas hasta miradas fundamentalmente biomédicas. Sin embargo, esta pluralidad de respuestas a cómo brindar un servicio público fundamental para el bienestar de las personas no se distribuyen de forma homogénea. Actualmente, el modelo hegemónico en el contexto español y catalán se encuadra en una aproximación psiquiátrica biomédica y psicofarmacológica globalizada, que articula una forma específica de androcentrismo y de relaciones de poder patriarcales en el campo de la salud mental como señalan Pla, Donat y Berna$\mathrm{beu}^{(2)}$. Y estos modelos de atención están cada vez más organizados según la gestión mercantilizada y un marco general de precarización financiera de los servicios públicos que dan la atención.

Los determinantes socioeconómicos y su efecto en el tratamiento de la salud mental han sido ampliamente estudiados, pero recién con el impacto de la crisis de 2008 se han comenzado a realizar importantes trabajos en esta línea. El factor socioeconómico es el mayor determinante en el aumento de la prevalencia de problemas de salud mental $^{(11,12)}$. Estos informes gubernamentales establecen que en el ámbito de la salud mental es donde hay más influencia del factor socioeconómico como determinante del bienestar de las personas. Han aumentado tanto la petición de tratamiento y el uso de servicios como el consumo de psicofármacos. Los diagnósticos de depresión, trastornos de ansiedad y problemas de sueño han sido los que más han crecido en España, principalmente, entre la clase trabajadora(13,14). 
Dado el incremento de demanda de atención y servicios de salud han aparecido nuevos estudios sobre la asistencia feminizada en salud mental y la situación de los y las profesionales sanitarias respecto a estos cambios. Predominantemente, se tratan de análisis de tipo cuantitativo y biomédico en los que, a modo de estudios epidemiológicos, se ven los efectos de la precarización de los servicios en la salud de las y los profesionales de la sanidad ${ }^{(8,15,16)}$. Estos estudios conceptualizan la enfermedad como un fenómeno individual y cuantificable, y traducen los malestares derivados de la situación profesional en un diagnóstico de burnout ${ }^{(17,18,19,20)}$. Concretamente, en el campo de profesionales de la salud mental, Lanham et al. ${ }^{(21)}$ y $\mathrm{O}^{\prime}$ connor et al. (22) muestran que son un colectivo especialmente expuesto, pero se hace sin datos desagregados por sexo y género. Aunque sea a nivel cuantitativo estas investigaciones ya nos dan indicios de los contextos de vulnerabilidad en los que se desarrolla la atención sanitaria en estos momentos.

Entre los trabajos con perspectiva crítica en torno a la atención en salud mental, encontramos dos principales focos de atención. Por un lado, hay un conjunto de estudios que han trabajado la vinculación del neoliberalismo y la atención en salud, investigaciones que representan una crítica profunda al modo en que se atiende la salud mental a raíz de la desmanicomialización de la década de 1980 y otras transformaciones contemporáneas. Vaccari ${ }^{(23)}$, Gallo Acosta y Quiñones Useche $^{(24)}$, Castro ${ }^{(25)}$, y Verhaeghe y Bracke ${ }^{(26)}$ ofrecen una rigurosa radiografía de cómo se ha dado este proceso en el contexto neoliberal, desde una mirada crítica que señala las consecuencias que ha tenido en las personas diagnosticadas. En el territorio español, Disviat $^{(27,28)}$ realiza un análisis de las potencialidades y las limitaciones de la reforma psiquiátrica a raíz de su 25 aniversario. También encontramos un interés incipiente sobre los derechos y la mejora del trato de las personas con diagnóstico en salud mental, impulsado por la Convención de los Derechos de las Personas con Discapacidad de Naciones Unidas ${ }^{(29)}$. En esta línea, actualmente emergen trabajos impulsados tanto por profesionales como por movimientos sociales de salud mental $\left.\right|^{(30,31,32,33,34)} \mathrm{O}$ de las personas supervivientes de la psiquiatría, por ejemplo. Por otro lado, hay una importante línea de trabajo con mirada de género en la que se problematizan los estereotipos y prejuicios de género ${ }^{(2,35)}$ y la feminización tanto de profesionales como de usuarias en el contexto de la relación asistencial ${ }^{(36)}$. Vinculado a los estudios de corte feminista hay trabajos ${ }^{(37,38,39)}$ que se acercan a nuestro objeto de estudio al introducir el concepto de cuidado en el contexto de la atención en salud mental.

En el presente trabajo entendemos a las y los profesionales de la salud mental a partir de la perspectiva de género en salud ${ }^{(40,41,42)}$, prestando especial atención a los estudios feministas de la ética del cuidado, aplicados a las relaciones profesionales en salud, más allá de las relaciones familiares ${ }^{(43)}$.

Hemos visto cómo, en el campo de la salud mental, predominan los trabajos de tipo epidemiológico y estadístico que entienden la salud como un fenómeno individual. Este tipo de trabajos nos aportan un conocimiento muy limitado de los factores sociales, culturales y relacionales de los contextos de vulnerabilidad. Con ese tipo de investigaciones no podemos hacer inteligibles los significados contextuales que las y los profesionales de la salud atribuyen a los conceptos de cuidado, malestar o de autonomía de la persona usuaria, por poner algunos ejemplos. Dichos conceptos son fundamentales en el día a día de la relación de atención, especialmente, en un momento histórico en el que tantas personas se encuentran en situación de sufrimiento psíquico, que aumentará exponencialmente en el marco de la pandemia de Covid-19, es de vital importancia conocer cómo se atiende y en qué condiciones lo hacen las y los profesionales, para poder favorecer una mejoría de los servicios. De ahí la relevancia como criterio de calidad de esta investigación, en el sentido de Calderón ${ }^{(44)}$. 


\section{Vulnerabilidad, ética del cuidado y salud mental}

Nuestro marco conceptual se apoya en los conceptos de contextos de vulnerabilidad ${ }^{(45)}$ y ética del cuidado ${ }^{(35)}$ para analizar las transformaciones que se están produciendo en la práctica profesional feminizada del personal de atención en salud mental. Cuando hablamos de contextos de vulnerabilidad adoptamos una posición teórica ${ }^{(13,14,45,46)}$ que entiende la vulnerabilidad como resultado de los aspectos relacionales y dinámicos de la vida social y no como un atributo de naturaleza individual, reducible a un rasgo biopsicológico. Consideramos que la vulnerabilidad viene determinada por las desigualdades sociales de género, de clase, de etnia, de edad, de capacidad, etc., que caracterizan nuestra sociedad. Los servicios públicos de atención en salud mental atienden diariamente a personas cuyas necesidades derivan de haber experimentado contextos de vulnerabilidad especialmente adversos y que han tenido una experiencia subjetiva particular de especial sufrimiento psíquico. El cuidado es una de sus principales necesidades a atender por parte de las y los profesionales de la salud mental, entendiendo el cuidado como

\footnotetext{
...un tipo de relación social que se caracteriza por la acción de satisfacer las necesidades de una persona por parte de otra, siendo el fin de la acción y donde la interacción cara a cara entre persona cuidadora y persona cuidada es un elemento crucial en el conjunto de la actividad. La necesidad es de tal naturaleza que no hay ninguna posibilidad de que pueda ser satisfecha por la persona objeto de cuidado. ${ }^{(47)}$
}

En este articulo exploramos algunos aspectos específicos de las relaciones de cuidado. De forma particular nos fijamos en dos dimensiones: la instrumental, es decir, aquellas acciones concretas y materiales que se emprenden con relación a los cuerpos; y la humana, el factor inmaterial relacionado con el vínculo afectivo-relacional y la interdependencia que se establece entre la persona cuidadora y la persona cuidada ${ }^{(40,41,43)}$.

Como desarrollaremos más adelante, todas las personas experimentan contextos de vulnerabilidad distintos y, por lo tanto, todas son susceptibles de necesitar cuidados. Las posiciones de cuidador, de cuidadora y cuidado no son fijas ni completas. Si se fijan, se corre el riesgo de que la persona que cuida genere sentimientos de omnipotencia (conIlevando una negación de la otra persona) y de resentimiento por parte de la persona cuidada, ya que puede generar dependencia o la idea de que el cuidado que recibe es una expresión de poder ${ }^{(48)}$. Una política democrática del cuidado es necesario que se funde en el reconocimiento recíproco y esta forma de abordar las actividades y las relaciones humanas la concebimos como una ética, un marco desde el que las personas toman consciencia de su situación de interdependencia.

El concepto de ética del cuidado surge de los trabajos sobre el desarrollo moral de Carol Gilligan ${ }^{(47)}$, en los que plantea una crítica a la idea de un desarrollo moral universal basado en la ética del derecho masculina. Se trata de un concepto que se ha utilizado como herramienta de análisis para estudiar relaciones sociales con perspectiva de género $^{(49,50,51,52)}$. Definimos el concepto de ética del cuidado como el modo de relacionarse con la vulnerabilidad ajena y la propia, que se aleja de aplicar soluciones universales, descorporeizadas y unilaterales. Su razón de ser se fundamenta en la lógica de la red y de la responsabilidad compartida ${ }^{(43,53)}$. Por lo tanto, a diferencia de la ética de la justicia (o del derecho) en ningún caso se asienta en individualidades aisladas y autosuficientes. Defiende una percepción de la moral como una red interconectada y compleja, en la que se entienden los conflictos en términos relacionales. Hace énfasis en la satisfacción de las necesidades humanas, valora el contexto y las diferencias existentes en la sociedad.

Para pensar el papel que puede jugar esta ética en la atención en salud mental, nos han interesado las implicaciones que tiene a nivel de la ciudadanía que configura. La ética del cuidado está estrechamente vinculada 
a un concepto de ciudadanía que rehúye la lógica liberal. El individuo (neo)liberal que presupone la ética de la justicia configura un modelo de ciudadano en concordancia con el imaginario del individuo universal y autosuficiente ${ }^{(54)}$, es decir, el de un hombre adulto, de clase media y occidental, cuya principal actividad es la profesional y en la esfera pública. Esta forma de entender la ciudadanía perpetúa la distinción publico/ privada, que confina los cuidados en la esfera privada y los asocia a la feminidad. Sin una subversión de este concepto de ciudadanía no se pueden democratizar los cuidados como ética fundamental en las relaciones humanas ni revertir la división sexual del trabajo. En la misma línea, Kohen ${ }^{(55)}$ lo expresa al afirmar que el desplazamiento a la esfera privada de las cuestiones relativas al cuidado ha sido una maniobra para sostener la ficción de que el ciudadano es autónomo, autosuficiente y que establece relaciones contractuales. Consideramos que desplazar la ética del cuidado a la esfera pública -en nuestro caso, a los servicios públicos de atención en salud mental- nos permite analizar si las prácticas clínicas se podrían entender como prácticas de resistencia respecto a las relaciones de desigualdad.

Nuestra propuesta de investigación es analizar qué papel tiene la ética del cuidado en la atención feminizada en salud mental a partir de los discursos de las y los profesionales y las personas usuarias sobre sus modos de entender la vulnerabilidad y de abordarla. En un contexto de conflicto capital-vida tan intenso, donde tantas personas ven cómo se agudiza su vulnerabilidad a nivel psicosocial y de imposibilidad de ejercicio de una ciudadanía digna y real ${ }^{(56,57)}$, sostenemos que es relevante estudiar la forma en que se da respuesta desde los servicios públicos de salud mental. Para ello, como hemos señalado, utilizamos el concepto de ética del cuidado como una herramienta conceptual con perspectiva de género que se fundamenta en la importancia de lo relacional y el apoyo mutuo como forma de actuar en el mundo. Así pues, nos preguntamos, para el caso estudiado, cuáles son los discursos que las y los profesionales en salud mental elaboran sobre la vulnerabilidad en la relación terapéutica entre profesional y persona usuaria, y si en estos discursos podemos identificar componentes de la ética del cuidado, así como sus implicaciones para un desplazamiento del concepto androcéntrico de ciudadanía.

\section{MÉTODO}

Como hemos señalado anteriormente, los resultados que exponemos en este artículo forman parte de un proyecto más amplio titulado La atención en salud mental desde una perspectiva de género: organización, gestión y subjetividad en el patriarcado neoliberal, dirigido por Enrico Mora y Margot Pujal de la Universitat Autònoma de Barcelona. El proyecto está organizado en dos fases: la primera consiste en un estudio de caso intensivo, en el sentido de Stake ${ }^{(58)}$, en un centro de salud mental. El estudio de caso es el dispositivo que se adecua mejor a las investigaciones que se proponen abordar un fenómeno complejo en su contexto real $y$, al mismo tiempo, prestar atención a un número limitado de eventos y condiciones y sus interacciones, lo que delimita la validez de los resultados aquí presentados. Una validez cualitativa, en el sentido de Calderon ${ }^{(44)}$, que no tiene por objetivo informar la calidad de esta investigación en términos de generalización estadística de los resultados, sino ofrecer un campo semántico de las diferencias que nos permita desarrollar un análisis en profundidad del fenómeno. La segunda fase consiste en una investigación-acción participativa, que pretende acompañar una transformación en pro del bienestar de las y los profesionales y la mejora de la atención a las personas usuarias. El presente artículo forma parte de la primera fase de estudio.

El diseño de investigación es abductivo, en el sentido de Vázquez ${ }^{(59)}$, mediante el cual llevamos a cabo una investigación que oscila entre la deducción (que va de lo general a lo particular) y la inducción (que va del resultado singular a lo general). La abducción es 
una aproximación que toma las preposiciones generales o abstractas como formulaciones provisionales, que se revisan en función de los resultados obtenidos, y que sirven de conjeturas para revisar las formulaciones iniciales y reorientar la obtención de resultados en un proceso reiterativo. Esta elección se adecua a la pregunta de investigación, cumpliendo con el criterio de adecuación epistemológica $^{(44)}$, en cuanto se trata de un campo sobre el que disponemos de muy poca evidencia empírica pero cuyos elementos teóricos no han sido aplicados en el campo de la salud. El diseño se llevó a cabo mediante el método discursivo ${ }^{(60,61)}$. Este método nos permite acceder tanto a experiencias del pasado como a expectativas de futuro respecto a la atención en salud mental; profundizar en pensamientos, percepciones y opiniones en tanto actores relevantes en dicho contexto; y conocer situaciones significativas, pero de difícil acceso, como lo es la consulta.

\section{Técnica de producción de datos}

Se escogió el grupo reflexivo, que se trata de un grupo de discusión, pero con la particularidad de que se busca indagar en la experiencia colectiva, la reflexión comunitaria y la introducción de la historicidad para comprender los procesos de cambio. Concretamente los grupos de los que se nutre este trabajo son cuatro: tres grupos de profesionales y un grupo de personas usuarias de los servicios de salud mental del centro estudiado. Su elección se debe a diversas razones: la característica relevante de esta técnica es poder aprovechar la discutibilidad que genera el grupo para poder obtener gran cantidad y variedad de información ${ }^{(60,61)}$; asimismo, la potencialidad que genera la situación grupal para hablar de vivencias duras o vinculadas a los colectivos concretos de los participantes; $y$, de cara a la segunda fase de la investigación-acción participativa del proyecto, esta técnica nos sirve para fomentar el sentimiento de comunidad y de cooperación tanto entre las y los profesionales y personas usuarias como con el equipo de investigación.

\section{Contexto del caso y participantes}

El ámbito en el que se ha buscado el centro de salud mental ha sido el área metropolitana de Barcelona. Allí se concentra la mayor parte de personas usuarias y la mayoría de los recursos de atención en salud mental de Catalunya. Los datos se han producido en un centro de salud mental de provisión pública en un distrito de Barcelona. Sin dar más detalles para mantener el anonimato, el centro que ha participado en la investigación es uno de los que más demanda de atención tiene y que nos ha permitido el acceso a su vida cotidiana profesional y de atención. Se trata de un centro con una tradición psicodinámica y comunitaria que, progresivamente, ha tenido que adaptarse al dispositivo sanitario contemporáneo. Allí encontramos un centro de salud mental de adultos, un servicio de rehabilitación comunitario y un programa de servicios individualizados.

Después de una sesión de presentación del proyecto, los y las profesionales participaron en grupos de discusión, nucleados según el tipo de servicio que presta el centro y según sus inquietudes e intereses. El resultado final es que prácticamente la totalidad de las y los trabajadores del centro han participado en alguno de los múltiples grupos de discusión-reflexión que se realizaron. Para cada grupo se realizó una media de siete sesiones distintas. Los grupos contaron con la participación, por un lado, tanto de hombres como de mujeres y asistieron todos los perfiles profesionales que trabajan con las personas usuarias $y$, por otro lado, grupos no mixtos de profesionales (cuyos datos no son objeto de este artículo). Cada grupo se formó atendiendo a que hubiera una importante variabilidad de edad interna para introducir en el debate la historia vivida en el centro y facilitar un vínculo significativo intergeneracional sobre las experiencias y cambios experimentados en el ejercicio de la profesión en la institución. Constatamos que hay una complementariedad de un muestreo teórico y pragmático ${ }^{(62)}$. Es decir, la composición y características de las y los participantes que 
nos viene dada coinciden con los requisitos a nivel teórico que le exigimos a la muestra.

Los grupos resultantes aglutinaron las profesiones relacionadas con la psicología clínica y terapias psicológicas, psiquiatría, enfermería, educación social, trabajo social y servicios administrativos. Nos hemos enfocado en los dos grandes servicios que ofrece la institución estudiada: el centro de salud mental de adultos y el servicio de rehabilitación comunitaria.

En el diseño de investigación incluimos también a las personas usuarias, como agentes claves en la relación asistencial, de manera de poder comprender mejor el ejercicio profesional de las y los profesionales. Para la configuración de estos grupos, se organizaron por sexo para crear un espacio facilitador y de confianza para las personas usuarias. La composición interna de cada grupo era variable respecto de la edad, el historial de cronificación y la duración del vínculo con los servicios de salud mental recibidos (Tabla 1 ).

Tabla 1. Número de participantes en los grupos de discusión. Barcelona, España. Noviembre de 2018 a abril de 2019.

\begin{tabular}{|lccc|}
\hline Participantes & Grupo & $\begin{array}{c}\text { Número de } \\
\text { mujeres }\end{array}$ & $\begin{array}{c}\text { Número de } \\
\text { hombres }\end{array}$ \\
\hline Profesionales & GDc1 & 6 & 3 \\
\hline GDc2 & 7 & 2 \\
\hline GDs & 3 & 2 \\
\hline Usuarias/os & GDu & 14 & - \\
\hline Total & & 30 & 7 \\
\hline
\end{tabular}

Fuente: Elaboración propia.

GDC1= Grupo de discusión 1 del centro de salud mental de adultos; GDC2= Grupo de discusión 2 del centro de salud mental de adultos; GDs=Grupo de discusión del servicio de rehabilitación comunitaria; GDu= Grupo de discusión de usuarias/os.

A efectos de los resultados aquí expuestos, hemos considerado los tres grupos de discusión con profesionales y el grupo formado por mujeres usuarias. Esto se debe a que solo en el grupo de mujeres usuarias hemos podido identificar aspectos que remiten a la ética del cuidado en relación con la práctica asistencial recibida. No creemos que esto signifique que el grupo de hombres usuarios no hayan participado de un vínculo regido por la ética del cuidado. Sin embargo, no disponemos de los datos para poder afirmarlo y describirlo.

Los temas generales a través de los cuales organizamos los grupos de discusión en sus diversas sesiones fueron: la historia del servicio de atención y del centro en el que desarrollan su actividad, las tipos de roles profesionales y formas de llevar a cabo la práctica clínica, la percepción de las personas usuarias, los ideales de feminidad y masculinidad en la relación terapéutica, la división sexual del trabajo profesional, las necesidades y demandas de profesionales y personas usuarias, las relaciones de poder entre las y los profesionales, y entre estos y las personas usuarias, y la incorporación de los derechos de las personas usuarias en el ejercicio profesional. Estas temáticas las abordamos desde las dimensiones normativas, fácticas y desiderativas.

Para el análisis, las sesiones fueron grabadas en audio. Se realizó una transcripción de los grupos para su posterior codificación, atendiendo a los objetivos de investigación. A efectos de las citas reproducidas en este artículo hemos realizado una corrección estilística sin alterar ni afectar el sentido original de lo enunciado. Esta corrección la realizamos como una forma de respeto a quienes han participado en los grupos, puesto que el registro oral es distinto al escrito, y produce en muchas ocasiones sentimientos de incomodidad para las personas participantes leer literalmente lo que han expresado oralmente. Asimismo, hemos omitido algunos detalles que no alteran el análisis pero que refuerzan nuestro compromiso de anonimato.

En cuanto a las consideraciones éticas, el tipo de estudio realizado fue aprobado por la institución que presta los servicios analizados, mediante convenio, así como por la asociación de personas usuarias referente del centro, en el que se establecieron los mecanismos de anonimato y consentimiento. Así, en la primera sesión de presentación del proyecto a las personas participantes, describimos el proyecto, leímos la hoja del consentimiento informado, y sus derechos, señalamos las garantías de anonimato y de custodia de los datos producidos en las instalaciones de 
la Universitat Autònoma de Barcelona. Después de un tiempo prudencial de reflexión, las personas que desearon participar en la investigación firmaron el consentimiento, que está también resguardado en la universidad.

\section{Procedimiento de análisis}

Se realizó un análisis de contenido-categorial o análisis temático que tiene por objetivo explicar y sistematizar el contenido de diferentes tipos de mensajes comunicativos, en nuestro caso, transcripciones de grupos de discusión. En palabras de Braun y Clarke ${ }^{(63)}$ este proceso consiste en identificar y analizar patrones dentro de los datos. Agrupando y organizando en forma de diferentes categorías temáticas los datos obtenidos; el objetivo ha sido profundizar en su contenido latente, relacionarlo con nuestro marco teórico propio y, en definitiva, con un contexto social e histórico ${ }^{(64,65)}$.

En primer lugar, se organizó el material a analizar y se buscó clarificar los objetivos del análisis. De ahí pasó a la fase de codificación en la que se transformaron los datos

Tabla 2. Categorías y subcategorías de análisis.

\begin{tabular}{|c|c|}
\hline Categorías & Subcategorías \\
\hline \multirow[t]{2}{*}{$\begin{array}{l}\text { Contextos de } \\
\text { vulnerabilidad }\end{array}$} & $\begin{array}{l}\text { 1. Determinantes contextuales del malestar } \\
\text { - Socioeconómicos } \\
\text { - Relacionales }\end{array}$ \\
\hline & $\begin{array}{l}\text { 2. Malestar derivado de la atención } \\
\text { - Estigma } \\
\text { - Vulneración de derechos } \\
\text { - Alteridad }\end{array}$ \\
\hline \multirow[t]{4}{*}{$\begin{array}{l}\text { Propuestas de abordaje } \\
\text { de la vulnerabilidad }\end{array}$} & $\begin{array}{l}\text { 1. Cuidado profesional } \\
\text { - Vínculo } \\
\text { - Relación terapéutica }\end{array}$ \\
\hline & $\begin{array}{l}\text { 2. Cuidado mutuo profesional } \\
\text { - Organización del trabajo } \\
\text { - Apoyo mutuo entre profesionales }\end{array}$ \\
\hline & $\begin{array}{l}\text { 3. Modelo comunitario } \\
\text { - Intervención ecológica } \\
\text { - Intervención integral } \\
\text { - Activismo en primera persona }\end{array}$ \\
\hline & $\begin{array}{l}\text { 4. Modelo hospitalario } \\
\text { - Medicación } \\
\text { - Atención hospitalaria } \\
\text { - Paternalismo }\end{array}$ \\
\hline $\begin{array}{l}\text { Precariedad del cuidado } \\
\text { profesional }\end{array}$ & $\begin{array}{l}\text { - Individualismo asistencial } \\
\text { - Presión asistencial } \\
\text { - Falta de recursos } \\
\text { - Necesidad de formación }\end{array}$ \\
\hline
\end{tabular}

brutos en unidades de sentido para permitir una descripción precisa de su contenido y terminar con la fase de categorización. El objetivo de la categorización es organizar y agrupar los códigos obtenidos utilizando criterios de similitud y diferencia para poder obtener una visión general de los datos. El resultado final refleja dos procesos diferentes de conformación de categorías. Por un lado, vemos cómo los mismos datos responden provisionalmente a aquellas preguntas que nos formulamos. Y, por otro lado, hay categorías que se han construido gracias a un proceso inferencial a partir de la organización de códigos con un contenido más latente que descriptivo que hemos ido revisando a partir de la estrategia abductiva antes descrita.

\section{RESULTADOS}

Fruto del proceso de codificación construimos un total de 19 códigos que las y los participantes relacionan con la atención en salud mental. A partir del proceso que hemos detaIlado hemos elaborado tres temas o categorías principales: contextos de vulnerabilidad, propuestas de abordaje de la vulnerabilidad y precariedad del cuidado profesional. Algunas de ellas desglosadas por subcategorías que ayudan a profundizar, ordenar e identificar elementos que constituyen el concepto de la categoría general (Tabla 2).

\section{Contextos de vulnerabilidad}

En esta categoría encontramos aspectos que usuarias y profesionales entienden que son fundamentales para entender el origen $y$ mantenimiento de situaciones de vulnerabilidad en relación con la salud mental. Ambos grupos se sitúan lejos del determinismo biológico de los genes y los neurotransmisores para explicar el sufrimiento psíquico. Vemos que dan un papel fuerte a las explicaciones de tipo socioeconómicas, en las que se vinculan de forma directa la falta de recursos mínimos para una vida digna (vivienda, trabajo 
estable, etc.) como raíz contextual de la vulnerabilidad de las personas usuarias y de su malestar psíquico. Por otro lado, otorgan importancia al hecho de disponer de buenas relaciones familiares y redes de apoyo como factores protectores del bienestar psicosocial.

Que hay personas que necesitan mucho tiempo, porque hay situaciones muy graves. No porque uno tenga un diagnóstico de esquizofrenia... No, no. Porque hay situaciones vitales que son gravísimas, porque hay situaciones de mucha violencia, precariedad económica. (GDs, profesional C1)

Sin embargo, este punto de confluencia entre lo que expresan las usuarias y las y los profesionales sobre buena parte del origen del sufrimiento psíquico, no se produce cuando en el discurso identificamos el malestar derivado de la atención. De ello hablan solo las usuarias, en términos biográficos, y alcanza todos los períodos en que se han encontrado, en alguna medida, vinculadas con los dispositivos de atención en salud mental, que incluyen épocas, establecimientos y profesionales muy diferentes. Un ejemplo paradigmático de dicho malestar derivado de los sistemas de salud mental es la revictimización institucional en casos de violencia de género. El paradigma biomédico dominante trata la violencia de género como un problema psicopatológico e individual, mediante el uso de psicofármacos, invisibilizándola como tal ${ }^{(66)}$ al carecer de una perspectiva de género interseccional centrada en la ética del cuidado y sin atender al contexto de desigualdad social que genera el sistema de género.

Las y los profesionales del establecimiento estudiado no expresan en su discurso relaciones entre la intervención clínica que desempeñan y si puede o no contribuir al malestar psíquico. Este, seguramente, es uno de los aspectos más espinosos de la práctica clínica y que más ambivalencia puede provocar en la reflexividad sobre la propia práctica terapéutica, en tanto la intervención está presidida por el principio de apoyar los procesos hacia el bienestar y nunca el de dañar. A pesar de ello, en el caso de estudio las y los profesionales han iniciado un proceso de cambio tanto en las dinámicas terapéuticas como en la forma de entender a las personas usuarias, que señala la voluntad y el deseo de transformación colectiva, el cual requiere de un tiempo y de un espacio de transformación reflexiva. Las usuarias expresan cómo, a lo largo de sus vidas, en ciertos momentos su relación con la red de servicios de salud mental de Barcelona ha tenido efectos perjudiciales o ha contribuido a mantener su malestar. Estas experiencias tienen que ver específicamente con la "vulneración de derechos", el "estigma" y la "alteridad". En cuanto a la vulneración de derechos, las usuarias se refieren a casos como el que explica la usuaria 2: "Hasta se me impuso un tratamiento que no quería". El estigma lo refieren, sobre todo, a la visión de las y los profesionales:

...y sobre todo el profesional no tener estigma. Porque hay muchísimos que tienen estigma. La forma de la que te ven. (GDu, usuaria 6)

Finalmente, respecto a la "alteridad" nos referimos a situaciones en las que se ha producido una relación de poder, ejercida en algunos casos por profesionales respecto de las usuarias, generando incluso la sensación de deshumanización.

Como realmente se me vulneró todos los derechos y todo eran ingresos, ingresos y más ingresos eso directamente no ayudaba a mi mejoría [...] Entonces era un pez que se mordía la cola. (GDu, usuaria 2)

Pero yo lo único que quiero de mi profesional es que me trate como una persona, que me escuche y que no me trate como un número. (GDu, usuaria 2)

Al mismo tiempo que señalan esos efectos adversos para su bienestar, también expresan en relación con la actual institución que les atiende, su reconocimiento a cómo han cambiado las cosas y cómo han encontrado un lugar de reconocimiento. 


\section{Abordaje de la vulnerabilidad}

Las y los profesionales y las personas usuarias que han participado en los grupos reflexivos de la institución estudiada entienden que la forma de abordar el sufrimiento psíquico debe pasar de un modelo centrado en la hospitalización a uno comunitario. Sin embargo, esta transición de modelo no siempre es fácil, dado que supone introducir cambios significativos en los supuestos epistemológicos y clínicos del conjunto del sistema de atención en salud mental, y aún no cuenta con los recursos necesarios por parte de las administraciones públicas sanitarias. Para las personas participantes es central la forma en cómo las y los profesionales y las mujeres usuarias se vinculan en el ámbito de la atención sanitaria. Este vínculo lo hemos denominado "cuidado profesional", y remite a que ese vínculo debe regirse por el cuidado, como elemento central del vínculo. Tanto para profesionales como para las mujeres usuarias, la horizontalidad y el respeto son herramientas de buen cuidado.

$Y$ que me tenga en cuenta porque, a fin de cuentas, soy yo la que lo estoy padeciendo. (GDu, usuaria 2)

Yo siento que he ido cambiando. En el sentido de entender más el poder que tiene el otro en sí mismo, las capacidades y el riesgo de mi poder. (GDc2, profesional C2)

Y junto con la necesidad de establecer relaciones de cuidado entre profesionales y mujeres usuarias, también lo es fomentar y sostener el "cuidado mutuo profesional". Aquí se muestra cómo al ser una profesión con un componente emocional muy fuerte, las y los profesionales dan mucha importancia al apoyo mutuo que se brindan entre compañeros y compañeras, así como al hecho de acompañarse en situaciones complejas derivadas de su quehacer profesional. Pero cuidar y cuidarse no es un tipo de relación que se puede establecer en el vacío o como fruto de una voluntad individual desprovista de condicionamientos contextuales e institucionales. Es decir, la capacidad para cuidarse tanto a sí mismos y mismas como a las personas usuarias tiene precondiciones. Las y los profesionales reivindican unas condiciones laborales adecuadas, con una buena organización del trabajo, como imprescindibles para poder ejercer como cuidadoras y cuidadores.
Para mí, cuidado lleva otras cosas que igual no las estamos contando [...] un horario que te vaya bien, un salario que te compense, una carga de trabajo que sea llevadera. (GDc1, profesional A2)

Pero la centralidad del cuidado en la forma de abordar la vulnerabilidad no es compatible con cualquier modelo de intervención sanitaria. Las personas participantes lo señalan, diferenciando, según las experiencias profesionales o como personas usuarias, dos formas principales de abordaje que otorgan significados y valoraciones diferenciadas.

El "modelo comunitario" emerge en un espacio entre los cambios recientes en la atención y un horizonte al que profesionales y usuarias expresan que desean llegar. Desde la perspectiva comunitaria se busca la intervención ecológica a través de la vinculación del entorno relacional de la persona afectada, trascendiendo su individualidad. Como "intervenciones ecológicas" se entienden el trabajo con la familia de la persona usuaria, atención domiciliaria o las actividades que buscan fortalecer la red de relaciones con la comunidad.

Para mí el ideal de futuro sería incluir la gente de fuera, la gente de la calle. La comunidad, vaya. (GDs, profesional E3)
Han hecho partícipes a mi familia y enton- ces ellos han entendido mejor la situación y entonces el apoyo ha sido realmente correcto, positivo. (GDu, usuaria 7)

También se basa en la "intervención integral", entendida como la articulación con diferentes agentes e instituciones para abordar la vulnerabilidad desde distintas dimensiones 
y no solamente la clínica-individual. La co-terapia y la implicación de servicios sociales o asesoramiento laboral serían ejemplo de ello.

Y, además, debería ser una cosa de muchas administraciones eh. No solo de sanidad sino de Bienestar Social, del Departamento de Trabajo... (GDc1, profesional P6)

Paralelamente, junto con esta orientación comunitaria de la atención en salud mental se aboga por la escucha de la voz de las personas usuarias, invisibilizada históricamente. Esto representa un cambio importante que guía el incipiente cambio de prácticas de atención: de la jerarquía al reconocimiento mutuo. En definitiva, el modelo comunitario busca abrir el cuidado en salud mental a nuevos agentes y escuchar las voces silenciadas.

Por su parte, lo que narran las personas participantes sobre el modelo comunitario, lo hacen refiriéndose, a modo de contraste, con el "modelo hospitalario", que para las y los profesionales y las usuarias tiene aún un excesivo peso en la concepción de la atención y en las instituciones de la red de salud mental.

Yo estaba acostumbrada a trabajar en agudos y claro, pasar a primaria fue un cambio importante para mí. Venía de una escuela muy biologista y venir aquí me costó. (GDc2, profesional E1)

...yo estaba en subagudos y ella directamente me dijo que aquello no era un servicio para trabajar nada sino para estabilizar. (GDu, usuaria 6)

Para las personas participantes, el modelo hospitalario se sustenta a partir de "intervenciones paternalistas" en las que las y los profesionales deciden por las personas usuarias. Se busca la estabilización inmediata con una especial centralidad de la "medicación". Se destacan la rigidez, la jerarquía y la presencia masculina en este modelo de atención.
O que los hombres tenían más facilidad de trabajar en el hospital porque quizá cobran más. (GDc1, profesional P2)

Sí que es verdad que en agudos hay más hombres. (GDc1, profesional E1)

...yo estaba en subagudos y ella directamente me dijo que aquello no era un servicio para trabajar nada, sino para estabilizar. (GDu, usuaria 6)

\section{Precariedad del cuidado}

En los grupos reflexivos de las y los profesionales ha sido recurrente la expresión de límites y acciones que sobrepasaban a su control individual y que lo entienden perjudicial para lo que creen que es un buen cuidado. Con precariedad del cuidado nos referimos a los procesos sociales que provocan las dificultades con que se encuentran las y los profesionales para poder ejercer el cuidado. Barreras que encuentran al querer atender la vulnerabilidad de las personas desde distintas dimensiones y atendiendo también a las necesidades de las y los propios cuidadores y cuidadoras. Y esta precariedad se articula a través de cuatro ejes: "individualismo asistencial", "presión asistencial", "falta de recur$\operatorname{sos}^{\prime}$ y "necesidades de formación".

Tengo una lista de espera de 180 pacientes y hay gente que está esperando psicólogo aquí en [...] desde hace un año. (GDc2, profesional C2)

Quizás al principio cuando entre aquí, cuando empecé era más... no sé, pensaba más las cosas. O no sé... podía reflexionar más. Ahora es visita, te metes en el despacho y pom pom pom. (GDc1, profesional P1)

Las y los profesionales no logran trabajar conforme las necesidades que detectan ni con las herramientas que les gustaría. Identificamos dos dimensiones de estos límites o dificultades. En primer lugar, las y los profesionales 
expresan que viven una importante presión asistencial que les limita el tiempo a dedicar a cada persona usuaria y, a la vez, la falta de recursos de los servicios de salud mental favorece esta dinámica de saturación. Por otro lado, las demandas de una reorientación de la formación que reciben las y los profesionales y la individualización de la asistencia son hechos que más bien tienen que ver con los efectos que tienen las formas tradicionales de atención psiquiátrica. Una formación rígida que se funda en el individualismo asistencial y su sostenimiento. Lejos de guías clínicas y "supertécnicas" diagnósticas expresan que echan de menos formación en relación asistencial y trabajo comunitario.

\section{Antes nos metíamos más en la cueva. Cada uno en su despacho y haciendo su faena. ¿Y cómo afecta eso en el paciente? Pues eso es menos, peor calidad asisten- cial. (GDc1, profesional P6) \\ Que los profesionales que van entrando en la red estén formados en la relación asistencial más que en supertécnicas. (GDs, profesional C1)}

Este hecho lo entienden como un empobrecimiento de la calidad del cuidado profesional y del conjunto de la atención que se da por parte de la red de atención en salud mental.

\section{DISCUSIÓN}

A la luz de los resultados para las categorías contextos de vulnerabilidad y propuestas de abordaje de la vulnerabilidad, se ve un vínculo entre el concepto de ética del cuidado que proponemos y la aspiración comunitaria que recorre el servicio de salud mental que hemos estudiado. Empezando por el concepto de vulnerabilidad. Las personas participantes lo conceptualizan de forma que la raíz del malestar de la persona usuaria no es una alteración cerebral que requiera una intervención psicofarmacológica, sino que tal y como expresa la profesional C1 "son situaciones vitales que son gravísimas". A nivel socioeconómico queda muy claro cómo la dinámica de crisis y de desprotección de las personas y protección de los mercados que supone el modelo neoliberal ha dañado mucho la salud psicosocial de las personas, especialmente de las mujeres de clases trabajadoras. Tanto las y los profesionales como las mujeres usuarias han expresado está cuestión en la misma línea, así como las numerosos investigaciones que han estudiado la relación entre salud mental y precariedad económica ${ }^{(11,13,14)}$. En esta línea, el Consejo de Derechos Humanos de la Organización de las Naciones Unidas ha emitido recientemente una resolución en la que aboga por el fortalecimiento de las condiciones dignas de vida como política de prevención en salud mental ${ }^{(46)}$. La centralidad que adquiera la etiología contextual y vinculada a las necesidades de cómo se entiende la salud mental es característica de una perspectiva ética en la que el cuidado pasa por encima de la norma biomédica, individualista y androcéntrica.

En cuanto a las propuestas de abordaje de la vulnerabilidad, hemos encontrado resultados pensables desde la ética del cuidado y de la justicia. Es importante destacar cómo a pesar de tener muy interiorizado su rol de cuidadores y cuidadoras, las y los profesionales también hacen mención de necesidades propias como comunidad y a las formas colectivas basadas en el apoyo mutuo para una atención sostenible y de más calidad que elaboran para responder a ellas. La permeabilidad, explícita en algún fragmento, en la frontera cuidador/a-cuidado/a resulta muy sugerente. Que las y los profesionales se piensen como vulnerables y pongan en valor unas condiciones mínimas para ejercer su rol de cuidadoras y cuidadores (especialmente visible cuando hablan sobre la organización del trabajo de atención) construye una nueva representación que se enfrenta a la autonomía y autosuficiencia, que se presupone a la ciudadanía desde una perspectiva liberal y de la ética de la justicia. Cuando hablamos de ética de la justicia nos referimos a que los principios que rigen 
...los objetivos de las acciones no atienden a las necesidades singulares contextualizadas de cada persona, o si lo hacen es en términos instrumentales. El logro del objetivo de la acción es ciego a cualquier consideración ajena al propio objetivo. El objetivo es el fin. Para ello, el logro del objetivo se realiza en función de normas universales y/o por la fuerza. ${ }^{(43)}$

Por lo tanto, nuevamente a través de la ética del cuidado se muestra una forma de entender a las personas que participan en la relación asistencial cercana al reconocimiento de una interdependencia ontológica. Aquí también se rompe la norma de género que vincula vulnerabilidad con el género femenino y el espacio privado, pues tal y como expresa Tronto ${ }^{(67)}$, la práctica de la ética del cuidado no tiene que ver con vincular las tareas de cuidado a las mujeres en el espacio familiar, sino en entenderlas "como una actividad humana transversal para mantener y reparar un mundo común", es decir, sostener la vida.

Las y los profesionales de los grupos reflexivos expresan que, a pesar de las dificultades que se encuentran en el día el día, tienen una vocación de cuidado, de autocrítica y de respeto hacia la persona que atienden, que está lejos de ciertas concepciones de la figura de la o del psiquiatra demonizado dominante en algunos momentos y contextos o que a veces proyectamos desde ciertas perspectivas críticas. No sabemos si se trata de una forma de entender la práctica clínica específica del caso de estudio o si está más generalizada. Para ello sería necesario realizar un estudio extensivo.

Por otro lado, en cuanto a las propuestas de cuidado profesional y el modelo comunitario las y los profesionales han dado una especial importancia a la horizontalidad en el vínculo asistencial entre profesional y persona usuaria, el reconocimiento mutuo, y a la escucha de la voz de la persona usuaria. Es decir, el factor relacionado con el vínculo afectivo-relacional y la interdependencia que se establece entre la persona que cuida y la persona cuidada ${ }^{(56)}$, que es una de las característica de la práctica democrática del cuidado. Los resultados apuntan a que la construcción de un vínculo fuerte $y$, sobre todo, con una relación menos jerárquica contribuye a que las personas usuarias se sientan tratadas como personas. Por parte de las y los profesionales es un proceso de renuncia de privilegios y de replanteamiento de su forma de entender la profesión y de los propios malestares que atienden, pero es un hecho que se vive como una mejora de su quehacer asistencial que contribuye al bienestar y a reducir el sufrimiento psíquico. Para ello, el medio para poder sostener este tipo de vínculo y abordar la vulnerabilidad es, según las personas participantes, el modelo comunitario. Esta forma de atender pone en el centro del cuidado la mirada sobre los procesos contextuales que viven las usuarias y las relaciones sociales que producen o mantienen el sufrimiento psíquico. Propone un abordaje colectivo para rehacer el vínculo comunitario de la persona o compartir el cuidado con otras instituciones próximas. La idea principal es que, si el malestar se da por fenómenos complejos y relacionales situados en la comunidad, debe ser en ella donde se pueda desarrollar el trabajo para la recuperación. En consonancia con Grandón Fernández et al. ${ }^{(68)}$ y Rendueles ${ }^{(69)}$ las redes de vínculos comunitarios fortalecen también las intervenciones de prevención en salud mental. Vistos los datos, creemos que es imprescindible una atención integral, ecológica y participativa en la salud mental de nuestras comunidades.

Además, al tratarse de un colectivo de profesionales que se ha feminizado de manera progresiva desde la segunda mitad del siglo XX, en el proyecto marco de este estudio, debemos preguntarnos por la influencia de la perspectiva de género (aún en tensión con un patriarcado neoliberal) en la tendencia profesional emergente en este estudio, orientada hacia una democratización del cuidado en un espacio público y profesional clave, como el de la atención en salud mental. 


\section{CONCLUSIONES}

La perspectiva comunitaria facilita la comprensión compleja y dinámica de la construcción de la subjetividad y de sufrimiento, así como de su abordaje, cambiando la perspectiva que entiende a las personas como meramente enfermas para pasar a conceptualizarlas como ciudadanas. Un concepto de ciudadanía que se distancia del implícito asociado al concepto hegemónico: hombre, adulto, de clase media, sano y capaz de las fábulas liberales. Desde esta perspectiva, un ciudadano autónomo no es como se piensa desde la ética de la justicia. La autonomía que podemos construir los seres humanos, desde una perspectiva atenta a la reducción del sufrimiento evitable y sus fuentes de desigualdad social, se basa en nuestra existencia compartida ${ }^{(52)}$, vulnerable e interdependiente. Esto significa establecer un marco de relaciones democráticas que implica desexualizar las prácticas de cuidado, cuestionando el sexismo y la división sexual del trabajo que encubre la transferencia sistemática de bienestar y apoyo psíquico a las posiciones hegemónicas masculinas a costa de las demás personas.

En un contexto tan hostil como el actual, que la pandemia de Covid-19 ha enfocado y ha exacerbado, al mismo tiempo, hay autores y autoras que sostienen que es imposible ejercer una ciudadanía no liberal y con derechos en estas condiciones ${ }^{(54,70)}$. Compartiendo su análisis, sin embargo, creemos relevante pensar la intervención comunitaria en salud mental como una guía para caminar hacia una ciudadanía contraria a la lógica individualista, patriarcal, neoliberal y de mercado. Lejos del falso universalismo, la única característica universal que reivindicamos desde la ética del cuidado es la vulnerabilidad consustancial a la especie humana junto a la vulnerabilidad forjada por las desigualdades múltiples de nuestra sociedad y el papel central que tiene el cuidado en el camino a construir nuevas formas de entender y actuar en la sociedad tanto en el espacio privado como en el público-profesional.
El modelo hospitalario convencional, a pesar de ser teóricamente replanteado en la última reforma en salud mental ${ }^{(27,71)}$, en los contextos catalán y español sigue vigente en la práctica profesional, el cual se presenta como incompatible con el ideal propuesto por las y los profesionales y las usuarias de una atención basada en el reconocimiento mutuo y la comunidad. A pesar de que hemos detectado una clara orientación a la ética del cuidado como propuesta de abordaje de la vulnerabilidad en los discursos de las personas participantes, identificamos una contradicción nuclear en la articulación entre las formas de organizar la red asistencial y las prácticas profesionales que fomentan las diversas instituciones que intervienen. En ella se aglutina una diversidad de formas de atención y modelos organizativos que dificultan la posibilidad de llevar a cabo una atención comunitaria coherente tanto para la persona usuaria, como para las y los profesionales. Así, puede estar oscilando entre distintos dispositivos cuyas premisas y principios se contradicen y que pueden implicar pasar del reconocimiento y respeto mutuo a la cosificación diagnóstica, el borrado de los contextos sociales de precariedad y el silenciamiento de su voz, una contradicción que no solo se da por modelos diferentes de entender la salud mental. La dinámica de precarización de las esferas de la vida derivada del neoliberalismo está creando contextos de vulnerabilidad múltiples para amplias capas de la sociedad, sumado a la creciente mercantilización y privatización lucrativa de los servicios públicos y de la complicada y contradictoria situación de sus profesionales, supone una dificultad enorme y un gran esfuerzo llevar a la práctica cotidiana la ética del cuidado correctora de una ética de la justicia excluyente e insostenible. Porque si no, el riesgo es aplicar una política individualista que suponga que la relación de cuidado debe estar a cargo de las/ los profesionales, sin el apoyo organizativo e institucional del centro asistencial y de la red, siendo una de sus principales consecuencias el malestar profesional colectivo, su agotamiento emocional y físico y el estrés ético fruto de las contradicciones de la encrucijada del patriarcado neoliberal actual. 
A la luz de lo que apuntan los resultados de la investigación, las intervenciones de orientación comunitaria pueden ser herramientas potentes para guiar nuevas formas de relacionarnos con nuestra vulnerabilidad compartida y en cómo atenderla. Esta perspectiva se basa en una ética que se sitúa bajo la lógica de la complejidad de las necesidades humanas y que, por lo tanto, es antagónica a las intervenciones existentes limitadas a la psicofarmacología y a los centros hospitalarios. El modelo comunitario promueve el reconocimiento de la persona más allá de su condición de enferma. Recupera su condición de persona y ciudadana a diferencia de lo que habitualmente han hecho las instituciones hospitalarias más tradicionales y todavía vigentes. Por ello, es fundamental el trabajo en red y la movilización de recursos colectivos en los barrios y los centros de salud, así como profundizar la reorganización de la propia red asistencial.

\section{REFERENCIAS BIBLIOGRÁFICAS}

1. Departament de Salut. Pla Director Sociosanitari 2006. Barcelona: Generalitat de Catalunya; 2006.

2. Pla I, Donat AA, Díaz IB. Estereotipos y prejuicios de género: factores determinantes en Salud Mental. Norte de Salud Mental. 2013;11(46):20-28.

3. Baranda L. La salut, els estil de vida i les condicons de treball dels pediatres a Espanya. Barcelona: Fundació Galatea; 2014.

4. Organización Mundial de la Salud. Promoción de la Salud Mental: Conceptos, evidencia emergente, práctica. Ginebra: OMS; 2004.

5. Organización Mundial de la Salud. Plan de Acción sobre Salud Mental 2013-2020 [Internet]. 2013 [citado 19 ene 2019]. Disponible en: https://tinyurl.com/ er3sb27s.

6. World Health Organization. Mental health atlas 2014. Geneva: WHO; 2015.

7. Espino A. Reformas de la salud mental en España. En: Salud mental en Europa: políticas y práctica Líneas futuras en salud mental. Barcelona: Ministerio de Sanidad y Consumo, Observatorio Europeo de Políticas y Sistemas Sanitarios; 2007. p. 489-532.
8. Ferlie E, Mcgivern G, FitzGerald L. A new mode of organizing in health care? Governmentality and managed networks in cancer services in England. Social Science \& Medicine. 2012;74(3):340-347.

9. de Vincent G, Mercier A. Manifeste pour sortir du mal-être au travail. París: Desclée de Brouwer; 2012.

10. Nogueiras Garcia B. La teoría feminista aplicada al ámbito de la salud de las mujeres: discursos y prácticas (España, 1975-2013). [Tesis doctoral]. Madrid: Universidad Complutense de Madrid; 2018.

11. Observatori del sistema de salut de Catalunya. Desigualtats socioeconòmiques en la salut i la utilització de serveis sanitaris públics en la població de Catalunya [Internet]. Agència de Qualitat i Avaluació Sanitàries de Catalunya; 2017 [citado 5 ene 2019]. Disponible en: https://tinyurl.com/kf3z3and.

12. Organisation for Economic Co-operation and Development. Health at a Glance: Europe 2018: State of Health in the EU Cycle [Internet]. OECD Publishing; 2018 [citado 10 ene 2019]. Disponible en: https:// tinyurl.com/mzj995rh

13. Arias-de la Torre J, Artazcoz L, Molina AJ, FernándezVilla T, Martín V. Inequalities in mental health in the working population of Spain: a National Health Surveybased study. Gaceta Sanitaria. 2016;30(5):339-344. 
14. London School of Economics and Political Science; Centre for Economic Performance, Mental Health Policy Group. The depression report: a new deal for depression and anxiety disorders. London: Centre for Economic Performance, London School of Economics and Political Science; 2006.

15. Brazil K, Kassalainen S, Ploeg J, Marshall D. Moral distress experienced by health care professionals who provide home-based palliative care. Social Science \& Medicine. 2010;71(9):1687-1691. doi: 10.1016/j.socscimed.2010.07.032.

16. Sejersted TS. Mortality among Norwegian medical doctors 1960-2000. BMC Public Health. 2011;11:173. doi: 10.1186/1471-2458-11-173.

17. Flórez J. Salud pública: El síndrome de burn out en médicos y enfermeras que atienden a pacientes oncólogicos. Medicina integral: Medicina preventiva y asistencial en atención primaria de la salud. 2000;36(10):405-411.

18. Gálvez Herrer M, Moreno Jiménez B, Mingote Adán JC. El desgaste profesional del médico: Revisión y guía de buenas prácticas. Madrid; Diaz de Santos; 2011.

19. Lacy BE, Chan JL. Physician Burnout: The hidden health care crisis. Clinical Gastroenterology and Hepatology. 2018;16(3):311-317. doi: 10.1016/j.cgh.2017.06.043.

20. Shanafelt TD, Noseworthy JH. Executive Leadership and Physician Well-being. Mayo Clinic Proceedings. 2017;92(1):129-146. doi: 10.1016/j.mayocp.2016.10.004.

21. Lanham M, Rye M, Rimsky L, Weill S. How gratitude relates to Burnout and job satisfaction in mental health professionals. Journal of Mental Health Counseling. 2012;34(4):341-354. doi: 10.17744/mehc.34.4. w35q80w11 kgpqn 26 .

22. O'connor K, Pitman S, Muller D. Burnout in mental health professionals: a systematic review and meta-analysis of prevalence and determinants. European Psychiatry. 2018;53:74-99. doi: 10.1016/j.eurpsy.2018.06.003.

23. Vaccari P. El fin del manicomio en tiempos del Neoliberalismo. [Tesis Doctoral]. Bellaterra: Universidad Autónoma de Barcelona; 2013.

24. Gallo Acosta JE, Quiñones Useche A. Subjectivity, mental health and neoliberalism in public health politics in Colombia. Athenea Digital: Revista de Pensamiento e Investigación Social. 2016;16(2):139-168.

25. Verhaeghe M, Bracke P. Associative Stigma among Mental Health Professionals: Implications for Professional and Service User Well-Being. Journal of Health and Social Behavior. marzo de 2012;53(1):17-32. doi: 10.1177/0022146512439453.

26. Castro X. Salud mental sin sujeto: Sobre la expulsión de la subjetividad de las prácticas actuales en salud mental. CS. 2013;(11):73-114.

27. Desviat M. La reforma psiquiátrica 25 años después de la Ley General de Sanidad. Revista Española de Salud Pública. 2011;85(5):427-436.
28. Desviat M. La Reforma Psiquiátrica. Valladolid: La Revolución Delirante; 2020.

29. Comité sobre los Derechos de las Personas con Discapacidad. Observaciones Finales sobre los Informes Periódicos Segundo y Tercero Combinados de España [Internet]. 2019 [citado 10 dic 2019]. Disponible en: https://tinyurl.com/2dz89hku.

30. Leamy M, Bird V, Boutillier CL, Williams J, Slade $M$. Conceptual framework for personal recovery in mental health: systematic review and narrative synthesis. British Journal of Psychiatry. 2011;199(6):445-452. doi: 10.1192/bjp.bp.110.083733.

31. Lolas F. Salud mental global como bien público: sugerencias para la reflexión ética. Revista de Neuropsiquiatría. 2013;76(3):131-136.

32. Pérez B, Eiroá F. Guia de drets en salut mental: no hi ha recuperació sense drets [Internet]. Federació Veus; 2017 [citado 10 dic 2019]. Disponible en: https://tinyurl. com/bv3rna2d.

33. Slade $M$, Amering $M$, Farkas $M$, Hamilton $B$, $O^{\prime}$ Hagan $M$, Panther $G$, et al. Uses and abuses of recovery: implementing recovery-oriented practices in mental health systems. World Psychiatry. 2014;13(1):12-20. doi: 10.1002/wps.20084.

34. Organización de las Naciones Unidas. Convención Internacional sobre los derechos de las personas con discapacidad [Internet]. 2007 [citado 7 dic 2020]. Disponible en: https://tinyurl.com/49f6p5bn.

35. Kortteisto T, Laitla M, Pitkäen A. Attitudes of mental health professionals towards service user involvement. Scandinavan Journal of Caring Science. 2018;32(2):681689. doi: $10.1111 /$ scs. 12495.

36. Oliveira MCP, Toassi RFC, Matos IB. Profissões e ocupações de saúde e o processo de feminização: tendências e implicações. Athenea Digital: Revista de Pensamiento e Investigación Social. 2013:13(2):239-244.

37. Amorim RG, Lavrador MCC. A perspectiva da produção de cuidado pelos trabalhadores de saúde mental. Psicologia: Ciência e Profissão. 2017;37(2):273-288.

38. Bruno Silveira C, Suelen Pontes Costa L, Bessa Jorge MS. Redes de atenção à saúde como produtoras de cuidado em saúde mental: uma análise reflexiva. Revista Portuguesa de Enfermagem de Saúde Mental. 2018(1):61-70. doi: 10.19131/rpesm.0203.

39. Pires RR, Ximenes VM, Nepomuceno BB. Práticas de cuidado em saúde mental no Brasil: análise a partir do conceito de cidadania. Avances en Psicología Latinoamericana. 2013; 31(3):507-521

40. Velasco S. Sexos, género y salud: Teoría y métodos para la práctica clínica y programas de salud. Minerva: Minerva Ediciones; 2009.

41. Pujal M. Apuntes para una salud mental inclusiva: duelo a la identidad de género y reconocimiento de la heterogeneidad de la experiencia. En: Climent Clemente 
MT, Carmona Osorio $M$, coord. Hacia una transpsiquiatría: abordaje queer de la salud mental y las relaciones de poder. Madrid: AEN Digital; 2018. p. 159-207.

42. Pujal M, Amigot P, Calatayud M. Subjetividad, desigualdad social y malestares de género. Una relectura del DSM5. Revista Internacional de Sociología. 2020;78(2):e155. doi: 10.3989/ris.2020.78.2.18.113.

43. Mora E, Pujal i Llombart M. El cuidado: más allá del trabajo doméstico. Revista Mexicana de Sociología. 2018;80(2):445-469.

44. Calderon C. Criterios de calidad en la investigación cualitativa en salud (ICS): apuntes para un debate necesario. Revista Española de Salud Pública. 2002;76(5):473482

45. Pujal M, Mora E. Contextualizar la vulnerabilidad: el diagnóstico psicosocial de género: El caso de la Fibromialgia. En: Montesó-Curto P, Rosselló Aubach L, coord. Compartir experiencias, combatir el dolor Una visión de la Fm desde el ámbito "bio-psico-social". Tarragona: Editorial de la Universitat Rovira i Virgili; 2017.

46. United Nations General Assambly, Human Rights Council. Right of everyone to the enjoyment of the highest attainable standard of physical and mental health [Internet]. 2019, Report No. 41 session [citado 15 may 2020]. Disponible en: https://tinyurl.com/yzu4wb9s.

47. Gilligan C. La ética del cuidado. Cuadernos de la Fundació Víctor Grífols i Lucas. 2000;30.

48. Izquierdo MJ. El cuidado de los individuos y de los grupos: ¿quién cuida a quién?: Organización social y género. Intercambios, Papeles de Psicoanàlisis / Intercanvis, Papers de Psicoanàlisi. 2003;(10):70-82.

49. Bellon E. Liderazgos femeninos: tránsitos hacia la ética del cuidado en las relaciones de género. Debate Feminista. 2017;54:84-100. doi: 10.1016/j. df.2017.03.002.

50. Burgos-Saelzer CB. Nursing care from the perspective of ethics of care and of gender. Investigación en Educación y Enfermería. 2013;31(2):243-251.

51. Castillo Cedeño I, Castillo Cedeño R, Flores Davis LE, Miranda Cervantes G. La ética del cuidado en la pedagogía saludable. Educación: Revista de la Universidad de Costa Rica. 2015;39(1):1-11.

52. García Moyano L. La ética del cuidado y su aplicación en la profesión enfermera. Acta Bioethica. 2015;21(2):311317. doi: 10.4067/S1726-569X2015000200017.

53. Izquierdo. Del sexismo y la mercantilización del cuidado a su socialización: Hacia una política democrática del cuidado [Internet]. 2003 [citado 10 dic 2019]. Disponible en: https://tinyurl.com/373rjuy8.

54. Cabanas E. La felicidad como imperativo moral: Origen y difusión del individualismo "positivo" en el capitalismo neoliberal y sus efectos en la construcción de la subjetividad. Madrid: Universidad Autónoma de Madrid; 2013.
55. Kohen B. Ciudadanía y ética del cuidado. En: Carrió E, Maffia D. Búsquedas de sentido para una nueva política. Buenos Aires: Paidós; 2005.

56. Orozco AP. Amenaza tormenta: La crisis de los cuidados y le reorganización del sistema económico. Revista de Economía Crítica. 2006;(5):7-37.

57. Rendueles C. César Rendueles: Este capitalismo es incompatible con la vida humana» [Internet]. Filosofía\&co.; 2018 [citado 20 dic 2019]. Disponible en: https://tinyurl. com/yx4a8xwe.

58. Stake R. Investigación con estudio de casos. Madrid: Ediciones Morata; 1999.

59. Vázquez L. La abducción como alternativa del método científico en la educación superior. Uni-Pluriversidad. 2009;8(2):1-12.

60. Elejabarrieta F. El método linguístico: técnicas de obtención de información. Barcelona: Universitat Autònoma de Barcelona; 1997.

61. Rolando PS, Víquez-Calderón D. Los grupos de discusión como metodología adecuada para estudiar las cogniciones sociales. Actualidades en Psicología. 2010;23 (110):87-101

62. Sanz J. La metodologia qualitativa en I'avaluació de polítiques públiques [Internet]. Institut Català de Polítiques Públiques; 2011 [citado 10 dic 2019]. Disponible en: https://tinyurl.com/2ppwdkak.

63. Braun V, Clarke V. Using thematic analysis in psychology. Qualitative Research in Psychology. 2006;3 (2):77-101.

64. Abela JA. Las técnicas de análisis de contenido: Una revisión actualizada [Internet]. Fundación Centro de Estudios Andaluces; 2002 [citado 10 dic 2019]. Disponible en: https://tinyurl.com/cb523paw.

65. Cáceres P. Análisis cualitativo del contenido: una alternativa alcanzable. Psicoperspectivas: Individuo y Sociedad. 2003;2(1):53-82. doi: 10.5027/psicoperspectivas-vol2-issue1-fulltext-3.

66. Tremoleda J. Suicidio y violencia de género: relación invisibilizada en los servicios de atención en salud mental. Mosaico: Revista de la Federación Española de Asociaciones de Terapia Familiar. 2019;(74):8-26.

67. Tronto JC. Moral boundaries: A political argument for an ethic of care. New York: Routledge; 1984.

68. Grandón Fernández P, Bustos Navarrete C, Tapia Cárdenas C, Cova Solar F. Evaluación de la restrictividad de dispositivos residenciales para personas con un diagnóstico psiquiátrico en el modelo de Salud Mental Comunitaria en Chile. Universitas Psychologica. 2015;14(4):1359-1370. doi: 10.11144/Javeriana.upsy14-4.epbs.

69. Rendueles C. Emancipación, cuidado y codependencia. Isegoría. 2014;(50):167-187. doi: 10.3989/isegoria.2014.050.09. 
70. Vidal-Molina P, Vargas-Muñoz R. Ciudadanía en tiempos del Capital: Una crítica desde la tradición marxiana. Convergencia. 2019;26(80):07. doi: 10.29101/ crcs.v26i80.10418.

71. Huertas R. De la reforma psiquiátrica a la salud mental colectiva: A propósito de la obra de Manuel Desviat. Revista de la Asociación Española de Neuropsiquiatría. 2020;40(138):163-170. doi: 10.4321/s0211573520200020009 .

\section{FORMA DE CITAR}

Martínez Flores J, Pujal i Llombart M, Mora E. Ética del cuidado y atención pública en salud mental: un estudio de caso en Barcelona. Salud Colectiva. 2021;17:e2966. doi: 10.18294/sc.2021.2966.

Recibido: 2 jul 2020 | Versión final: 23 dic 2020 | Aprobado: 22 feb 2021 | Primera publicación en línea: 22 mar 2021 | Segunda publicación en línea: 27 sep 2021

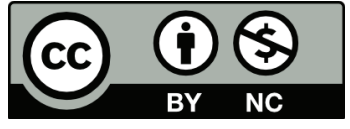

Esta obra está bajo una licencia de Creative Commons Reconocimiento-No Comercial 4.0 Internacional. Reconocimiento - Permite copiar, distribuir y comunicar públicamente la obra. A cambio, se debe reconocer y citar al autor original. No Comercial - Esta obra no puede ser utilizada con finalidades comerciales, a menos que se obtenga el permiso.

https://doi.org/10.18294/sc.2021.2966

\section{FE DE ERRATAS}

La primera versión publicada de este artículo contenía errores en la numeración de las referencias. Dado que esa primera versión ya había comenzado a circular, nos vimos en la obligación de despublicar el artículo, incorporar las correcciones y volver a publicarlo como una segunda versión. 